\title{
Le degré d'intégration des sous-traitants marocains dans le secteur du textile-habillement : est-il vraiment une sous-traitance de capacité ?
}

\author{
Khalid KHOURIBA $^{1}$ et Mohamed YAOUHI ${ }^{2}$ \\ ${ }^{1}$ Université Mohammed $V$ de Rabat, Maroc \\ ${ }^{2}$ Université Mohammed V de Rabat, Maroc
}

\begin{abstract}
Résumé : L'industrialisation des territoires est un processus dans lequel le Maroc s'est engagé depuis plusieurs années. Néanmoins, à partir des années 2000, le Maroc a mis, de plus en plus, l'industrie au centre de ses préoccupations, visant le développement d'une économie basée sur l'industrie à travers l'intégration des chaines de valeurs mondiales. Pour ce faire, plusieurs fonds et programmes d'accompagnement, d'assistance et d'appui des PMI, PME et des grandes entreprises ont été mis en place. A la lumière des autres secteurs industriels, le textile-habillement a enregistré des bons chiffres en matière de création d'emploi. Cependant, la vraie réussite se trouve dans le développement d'une industrie nationale compétitive et durable déclenchée par l'émergence d'unités industrielles nationales capables de se positionner dans les chaines de valeurs mondiales et dans la pyramide de la sous-traitance. Donc, de passer d'une simple sous-traitance de capacité tirée par le coût de la main à la fabrication de produits finis ou au moins à une sous-traitance de spécialité et d'intelligence. C'est pourquoi il a été jugé très pertinent de réaliser une enquête traitant le degré d'intégration des soustraitants marocains dans le secteur du textile-habillement.
\end{abstract}

Mots-clés : intégration, sous-traitance, capacité de production, donneurs d'ordres, chaine de valeur.

\section{INTRODUCTION}

La montée en puissance de la mondialisation et les stratégies de globalisation ont conduit à la restructuration des chaines de valeurs mondiales (CVM). De même, à cause du progrès technologique rapide, la mondialisation est toujours sur son chemin. Voire, la globalisation elle-même évolue en créant plus de déplacement de chainons vers des pays en développement notamment dans tous les domaines, y compris le secteur du textile-habillement (Mezouaghi et Aynaoui, 2018 ; Khallaf, 2009). Ce déplacement est le résultant de plusieurs facteurs dont les principaux sont : la main d'œuvre à bon marché, la connectivité, la proximité, la langue commerciale, les accords de libres échange, les conventions, les normes et standards, la stabilité, les avantages fiscaux, les infrastructures industrielles, technologiques et commerciales, etc. Bien que cette restructuration ait fait émerger de nouvelles niches pour les entreprises marocaines, elle a aussi engendré des défis et enjeux liés à leur compétitivité. En conséquence, les acteurs concernés ont dû mettre en place des solutions pour faire face à ces défis. Néanmoins, l'environnement des entreprises reste toujours caractérisé par l'incertitude, le changement perpétuel et la complexité. Ce qui interpelle des recherches mettant l'accent sur l'analyse et la compréhension des phénomènes aux temps opportuns afin d'en tirer profit ou de se protéger.

Bien que le textile-habillement ait émergé dès l'indépendance comme le premier pôle industriel du secteur privé (Bakal, 2017), il a subi des difficultés au cours de l'année 2005 qui ont touché aussi bien les exportations 
que l'emploi. Ainsi, les exportations ont chuté entre janvier et décembre 2005 de $10 \%$ à $15 \%$ et 40000 emplois ont été perdus (Khallaf, 2009). Cette crise a été déclenchée par la concurrence chinoise qui a dominé sur le marché international notamment par le prix qui a attiré les grands donneurs d'ordres européens. Ces derniers ont largement déplacés leurs commandes vers l'Asie. Par conséquent, les entreprises marocaines opérant dans ce secteur ont été gravement impactées, en particulier celles qui n'avait que le prix comme point fort.

Toutefois, le secteur du textile reste toujours une composante essentielle de l'économie marocaine. Ainsi, il a bénéficié du redimensionnement des chaines de valeurs depuis des années. Cependant, la prospérité de ses acteurs a été fortement menacée par la concurrence acharnée provoquée par plusieurs pays, notamment les asiatiques, une partie des pays de l'Europe de l'Est et la Turquie. Cette concurrence se manifeste principalement au niveau des prix, de la qualité et au niveau des délais de livraison et services annexes. Ce qui a conduit le gouvernement marocain, en partenariat avec les acteurs privés (notamment les associations professionnelles), à la configuration de nouveaux plans et stratégies, suivant une approche participative. Ainsi, des mesures incitatives et d'accompagnement ont été mises en place visant l'appui des entreprises opérant dans le secteur et l'attractivité de nouvelles entreprises, en se focalisant sur les chainons dans lesquels les entreprises marocaines sont compétitives. Ainsi, des efforts louables ont été déployés pour améliorer la compétitivité des entreprises opérant au Maroc.

Par conséquent, depuis l'entrée en vigueur de la nouvelle stratégie baptisée Plan d'Accélération Industrielle (PAI 2014-2020), le secteur du textile commençait à récolter les résultats de cette reconfiguration et redynamisation en matière de création d'emploi, d'exportation et d'implantation de nouvelles unités industrielles et commerciales (extensions et nouvelles créations). Toutefois, il est aujourd'hui le temps opportun de s'interroger sur le degré d'intégration des sous-traitants marocains opérant dans le secteur du textilehabillement.

2. REVUE DE LITTERATURE

\section{A. Sous-traitance dans les écosystèmes textile-habillement : un secteur qui trouve sa force dans la sous-traitance}

Si les écosystèmes automobiles et aéronautiques ont été dotés d'une intention particulière de la part des pouvoirs publics au Maroc, le secteur du textile-habillement lui aussi a bénéficié de sa part des mécanismes de développement, d'appui et d'accompagnement. Ainsi, plusieurs fonds et mécanismes de financement ont été mis en place ces dernières années permettant le renforcement des acteurs de ce secteur. Parmi ces instruments de financement, il figure le FIT, le FDII, FHII, etc. Via ces fonds, l'Etat a mis en place des packs et programmes d'appui des entreprises de différentes tailles (autoentrepreneur, PMI, PME et GE) et ce, pour accompagner les entreprises à enlever leurs difficultés liées au financement de leurs investissements (création de nouvelles usines, développement des systèmes de management, renouvellement des systèmes de production, etc.). A signaler que les contraintes financières se classent au premier niveau de difficultés que confrontent les entreprises opérant dans ce secteur (Chaffai et Plane, 2015). Dans le même sens, la Banque Mondiale a constaté en 2006 que sur l'ensemble du secteur de la fabrication, $80 \%$ des entreprises marocaines se sentent gravement affectées par des contraintes financières (World Bank, 2006). De même, l'Etat a créé des établissements et agences gouvernementaux, en plus du ministère chargé de l'industrie et de l'investissement, dont le développement du secteur du textile-habillement fait partie de leurs principales missions, notamment l'ANPME ${ }^{1}$ et l'AMDIE ${ }^{2}$. Ces

\footnotetext{
${ }^{1}$ Agence Nationale pour la Promotion de la Petite et Moyenne Entreprise.

${ }^{2}$ Née de la fusion de « l'Agence Marocaine de Développement des Investissements (AMDI) », du « Centre Marocain de la Promotion des Exportations (CMPE) » et de " l'Office des Foires et des Expositions de Casablanca (OFEC)», l'Agence Marocaine de Développement des Investissement et des Exportations (AMDIE) a été créée en décembre 2017 par la loi n 60 16.
} 
efforts ont abouti à l'augmentation de la capacité de production de ce secteur via l'implantation de nouvelles usines, soit sous forme d'une première création (création de nouvelles sociétés) ou extension des sociétés déjà existante (création de nouvelles usines).

A l'instar des autres secteurs industriels, l'industrie du textile au Maroc, a subi de fortes mutations engendrées par la mondialisation, la restructuration des chaines de valeur mondiales et la concurrence accrue émergée principalement par les asiatiques et les pays de l'Europe de l'Est (Httabou et Louitri, 2011). Sans oublier l'intensification des relations de la Chine avec l'Afrique (Hugon, 2008), et l'élargissement des pays bénéficiant de l'accès au marché européen (Belghazi, 2005).Ce qui impose au Maroc de renforcer sa diplomatie économique et appuyer ses entreprises (Dafir et Salam, 2016). Par conséquent, les entreprises installées au Maroc se sont retrouvées dans l'obligation de se focaliser sur les maillons de la chaine dans lesquelles elles sont compétitives. Même, les appuis des pouvoirs publics ont été orientés vers ces segments.

Les forces du secteur de textile se matérialisent par la disposition du Maroc de ses propres modèles. Par ailleurs, depuis sa segmentation en écosystèmes, le textile au Maroc commence à créer de la valeur ajoutée (Al Alami, 2017)3. De même, il s'agit d'un secteur orienté vers l'export en enregistrant une croissance de plus de 32\% entre 2012 et 2016 (AMDIE, 2017). Il emploie plus de 27\% des emplois industriels et contient plus de 1000 entreprises (MICEVN, 2017). A cet égard, il est caractérisé comme le secteur traditionnel du Maroc.

Depuis la mise en place du PAI, le secteur du textile a été segmenté en six écosystèmes tel que schématisé ci-après :

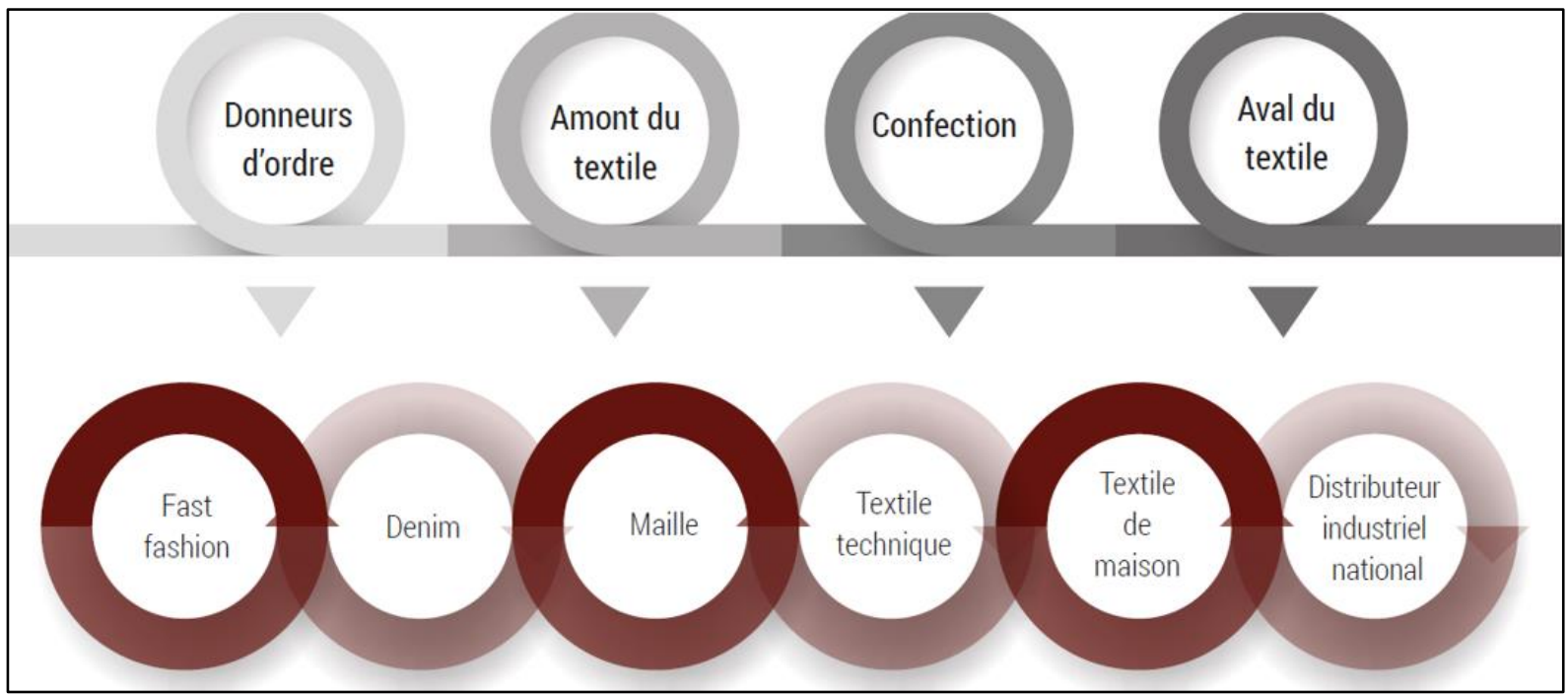

Figure 1 : organisation du textile en six écosystèmes

\section{B. Caractéristiques des relations de sous-traitance dans le secteur du textile}

A cause de la sous-traitance, le Maroc est devenu un champion de la Fast Fashion et partenaire international d'enseignes de référence et a réussi à devenir le troisième fournisseur textile pour l'union européenne (AMDIE, 2017), alors qu'il était le sixième avant la mise en place de la dernière stratégie industrielle (Httabou et Louitri, 2011). Bien qu'il reste toujours une composante clé de l'économie marocaine, une large partie de la production

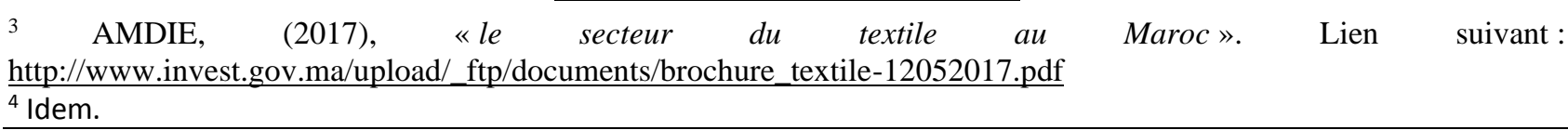


de ce secteur consiste à répondre aux attentes des donneurs d'ordres européens (Tazi, 20195 ; Piveteau, 2009). L'attractivité des donneurs d'ordres européens en premier niveaux, est due aussi, aux stratégies de ces grandes entreprises pour s'adapter aux nouveaux enjeux du secteur. Ainsi, les entreprises européennes se sont organisées en réseaux en optant de plus en plus pour les délocalisations (Ammar et Roux, 2009 ; Meddeb, 2000). Le Maroc, était parmi les meilleurs bénéficiaires de cette organisation des grands donneurs d'ordres européens, après avoir constaté l'obligation de s'arranger aux changements (Piveteau, 2009).

Plusieurs études ont constaté que les relations de sous-traitance dans ce secteur sont polarisées par les grandes entreprises exportatrices (Belghazi, 2005). Ces dernières représentent une interface des donneurs d'ordres étrangers, notamment européens. Elles créent des réseaux de sous-traitants locaux disposant d'une identité juridique et d'une certaine autonomie, mais dans la plupart des cas, rattachés au propriétaire unique. Ainsi, il pourrait être constaté qu'une entreprise exportatrice ne dispose que de quelques fonctions de support tandis que les ateliers de production sont organisés sous forme de sociétés disposant d'identités juridiques propres. La création de nouvelles unités a pour objectif de bénéficier des avantages données par la charte d'investissement ou pour doter ces unités d'une certaine autonomie en se procurant d'avantages organisationnels (Belghazi, 2005).

Selon Belghazi (2005), les relations de sous-traitance dans le secteur de textile au Maroc sont caractérisées par une diversité. Il a remarqué, aussi, qu'il existe des cas où le capital du sous-traitant est intégralement contrôlé par le donneur d'ordres et également des cas où aucune relation ne lie le sous-traitant avec le donneur d'ordres, sans parler des entreprises qui préfèrent de ne pas sous-traiter les commandes qu'elles reçoivent. Ainsi, Belghazi (2005) a constaté l'existence de deux modèles de relations de sous-traitance; le modèle de sous-traitance à relations stabilisées et le modèle de sous-traitance à relations distendues.

Comme son nom l'indique, le modèle de relations stabilisées est caractérisé par une certaine standardisation et l'instauration de normes de production strictes permettant le développement de la relation en continu. Quant au modèle de relations distendues, il est caractérisé par l'existence des clients qui sont des donneurs d'ordres et qui distribuent leurs commandes sur plusieurs dizaines d'opérateurs locaux propriétaires de leurs entreprises. Les relations de sous-traitance dans ce modèle sont régies par des contrats de courtes durées et marquées par l'instabilité (Belghazi, 2005) ${ }^{6}$. Cette classification a été faite selon la durée de la relation entre les sous-traitants et leurs clients. A noter que les clients (donneurs d'ordres) marocains sont aussi des sous-traitants de donneurs d'ordres généralement européens. Dans le cas des relations stables, et lorsqu'il s'agit d'un donneur d'ordres marocain (sous-traitant de premier rang), ce dernier crée des entités juridiquement indépendantes mais détient leurs capitaux, ce qui lui permet de les contrôler et assurer leur management et leur organisation. Dans une telle situation, le donneur d'ordres contrôle tous le circuit de production de la commande qui lui a été confiée. Il procède à l'adaptation de la capacité de production du groupe. Ces deux relations peuvent être associées à des impacts sur la nature de l'emploi comme l'a fait constater Belghazi (2005).

Toutefois, qu'elles soient liées par des relations stables ou distendues, liées à des donneurs d'ordres marocains ou étrangers, qu'en est-il de l'intégration des unités industrielles marocaines dans ce processus de sous-traitance?

\footnotetext{
${ }^{5}$ Lors du congrès international de l'Association Marocaine de Gestion à Marrakech les 20 et 21 juin 2019 sous le thème : les nouveaux défis de la compétitivité des entreprises.

${ }^{6}$ Pour plus de détails concernant ces deux modèles, voir Saâd Belghazi, (2005), «Pratiques des donneurs d'ordre et précarité du travail dans le textile habillement marocain », INSEA, Rabat, $\mathrm{p}: 6$ à 8
} 


\section{ProblematiQue Generale ET HYPOTHESE DE RECHERCHE}

\section{A. Problématique}

L'industrialisation des territoires est un processus dans lequel le Maroc s'est engagé depuis plusieurs années. Néanmoins, à partir des années 2000, le Maroc a mis, de plus en plus, l'industrie au centre de ses préoccupations, visant le développement d'une économie basée sur l'industrie à travers l'intégration des chaines de valeurs mondiale. Pour ce faire, plusieurs fonds et programmes d'accompagnement, d'assistance et d'appui des PMI, PME et des grandes entreprises ont été mis en place. En dépit de réels succès sectoriels, comme dans l'automobile ou l'aéronautique, le bilan insuffisant des deux premiers Plans et Pacte pour l'émergence industrielle a cependant conduit l'Etat marocain à ajuster son approche au travers du Plan d'Accélération Industrielle (PAI 2014-2020) (Piveteau, 2018). En 2014, une nouvelle stratégie, succédant le PNEI, a été lancée, après avoir été présentée devant SM le roi Mohamed VI, dénommée le Plan d'Accélération Industrielle (PAI). Le Maroc se veut de cette stratégie de passer à un autre niveau et donner un nouvel élan à l'importance de l'industrie dans l'économie marocaine. Le PAI a été construit à travers une approche participative basée sur les écosystèmes. Visant essentiellement la création d'emploi (500 000 emplois à l'horizon 2020), l'augmentation des exportations (équilibre de la balance commerciale) et l'augmentation du poids de l'industrie dans le PIB (augmentation de 9 points) en passant de $14 \%$ à $23 \%$.

En plus des incitations financières et fiscales, le gouvernement marocain a procédé à la modernisation et l'extension de son infrastructure en général, à savoir l'amélioration des infrastructures industrielles, technologiques, commerciales et logistiques. A cause de son offre globale compétitive, le Maroc a vu des opérateurs leaders s'installent chez lui, notamment dans le secteur du textile.

Cependant, la vraie réussite se trouve dans le développement d'une industrie nationale compétitive et durable déclenchée par l'émergence d'unités industrielles nationales capables de se positionner dans les chaines de valeurs mondiales et dans la pyramide de la sous-traitance. Ce positionnement peut être atteint à travers la soustraitance. Car les chaine de valeur mondiales sont contrôlées et dominées par des grandes entreprises (OCDE, 2013). Les capacités de production, managériales et d'innovation (organisation, technologie, logistique, positionnement, relationnelle, robotisation, etc.) de ces entreprises dépassent largement celles des entreprises locales. La figure suivante schématise ce postulat :

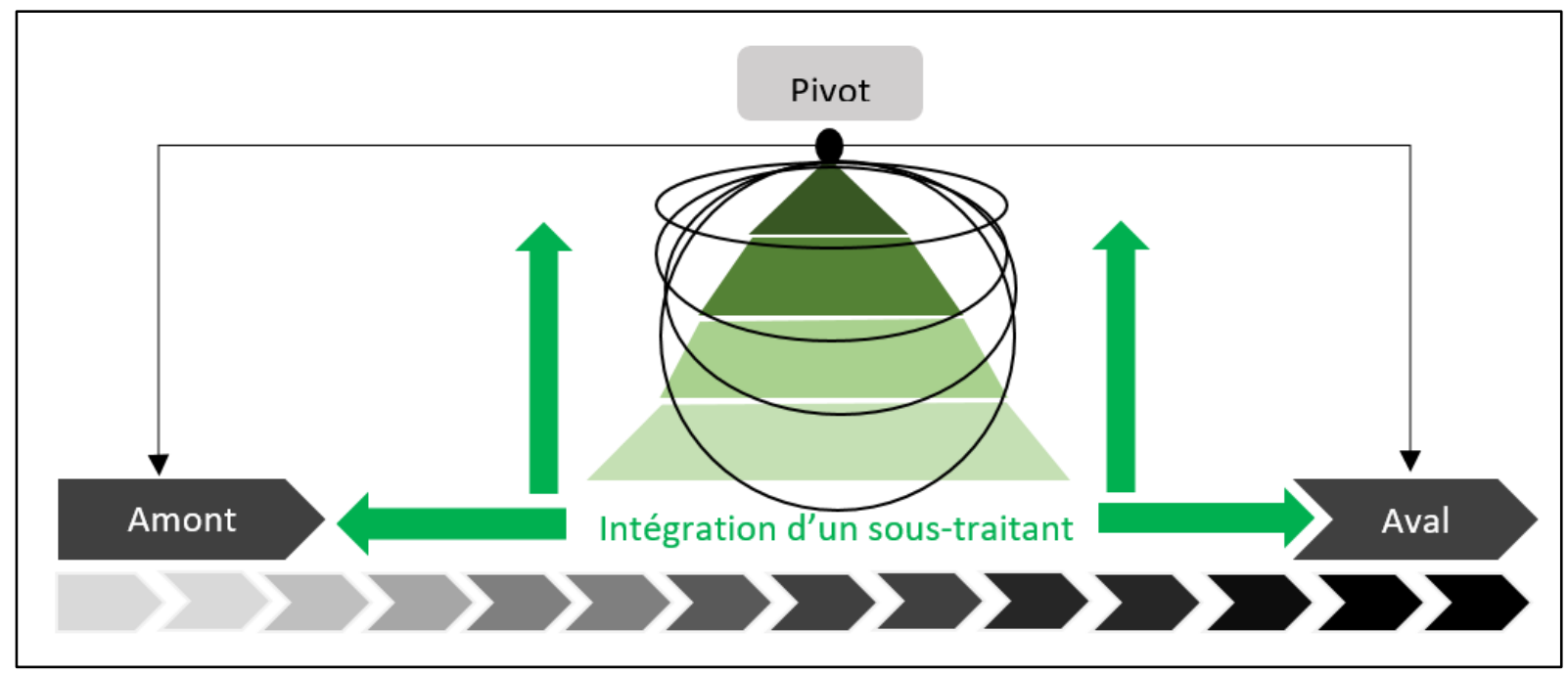

Figure 1 : l'intégration des unités industrielles locales dans le processus de sous-traitance 
Tel que présenté dans le schéma ci-dessus, l'amont et l'aval d'une chaine de valeur sont généralement trop contrôlés par le pivot (l'entreprise principale). Car, c'est là où se trouvent les chainons à forte valeur ajoutée et les clefs de la dominance. L'amont se compose des étapes de conception et de développement des produits et services et l'aval se compose des chainons de commercialisation et de développement de l'image de l'entreprise. Par conséquent, le contrôle de ce genre d'activités est indispensable pour la survie de n'importe quel pivot. C'est pourquoi, les grands donneurs d'ordre s'occupent souvent eux-mêmes des chainons en amont ou les délèguent à d'autres entreprises disposant de caractéristiques techniques dépassant les leur. C'est la raison pour laquelle, nous admettons que le passage au premier rang dans la pyramide de sous-traitance est une montée en puissance des capacités techniques et managériales du sous-traitant, soit un élément reflétant son niveau d'intégration.

Une fois une partie des activités ou tâches, réalisée auparavant par un donneur d'ordres ou une autre entreprise pour son compte, est confiée à un nouveau sous-traitant, il s'agit de son premier pas vers l'intégration de la chaine de valeur de son donneur d'ordres. Il commence parallèlement un autre processus d'intégration dans la pyramide de sous-traitance. L'évolution dans les rangs de sous-traitance est un élément révélateur de l'acquisition de nouvelles compétences. A ce propos, il convient de préciser que la classification des relations de sous-traitance en sous-traitance de capacité, de spécialité, de fourniture ou d'intelligence est fonction de la nature des prestations confiées au sous-traitant, de son degré de participation dans la conception des services ou produits qui lui sont confiés et de son importance dans le réseau de la grande entreprise (Billaudot et Julien, 2003 ; St-Jean et Lebel, 2010 ; Billaudot et Genthon, 2001 ; Renou, 2006 ; Benguerel, 1989 ; Clerwall, 2013 ; Altersohn, 1992 ; Baudry, 2013 ; Billaudot et Julien, 2003 ; Benabdeljlil, 2013 ; Benabdeljlil et al., 2017 ; Kechidi, 2006 ; Benhar, 2016 ; Carrincazeaux et Frigant, 2006 ; Mazaud, 2007).

De même, le sous-traitant commence à intégrer des chainons en allant vers l'amont et l'aval de la chaine de valeur. Une intégration totale correspond à la livraison de produits à son propre nom. Ce qui fait émerger aussi la relation entre le niveau d'intégration et les compétences des sous-traitants. Cependant, ces compétences sont influencées par les actions d'appuis externes entreprises par l'Etat, les associations professionnelles ou les entreprises partenaires. Dans ce sens, il convient de signaler que le mot qu'avons-nous utilisé dans l'intitulé de ce travail «processus » désigne le macro-processus de la sous-traitance. Il se compose de deux principaux processus ; un processus vertical relatif à la hiérarchisation des étapes pour se placer au premier rang et un processus horizontal relatif à l'intégration des maillons de la chaine de valeur du produit final en question. Bien sûre, ces deux processus peuvent être ventilés en sous-processus jusqu'on arrive aux activités et tâches.

Comme nous avons étayé en haut, plusieurs études ont porté, totalement ou partiellement, sur le processus de sous-traitance au Maroc. Toutefois, aucune de ces études n'a mis l'accent sur l'intégration des unités industrielles locales dans ce processus. Ce qui fait émerger la problématique suivante :

Les sous-traitants marocains opérant dans le secteur du textile ont-ils réussi à développer leur niveau d'intégration dans le processus de sous-traitance ou il s'agit seulement de sous-traitance de capacité tirée par le coût?

L'importance de cette problématique la rend indispensable d'être étudiée dans un cadre de recherche encadré par la théorie et vérifié par l'empirique à travers des enquêtes menées auprès d'un échantillon de sous-traitants.

\section{B. Variables de recherche et hypothèses de travail}

Le principal objectif de cette recherche est d'une part l'évaluation de l'intégration des sous-traitants marocains et d'autre part, l'identification des facteurs et paramètres qui contribuent de plus à l'évolution du niveau de ces entreprises. Quant à l'évaluation du niveau d'intégration, elle se fera à travers l'analyse des variables suivantes : 


\section{- Degré d'intégration (capacité de passage au produit fini)}

Le processus d'intégration via lequel est mesurée cette variable est composé de trois phases. Chaque phase correspond à un groupe d'entreprises. Le premier groupe est composé des entreprises qui sont à 100\% soustraitantes. Le deuxième groupe est composé des sous-traitants qui ont passés à la cotraitance. Quant au dernier groupe, il est composé des sous-traitants qui ont réussi à développer et vendre des produits finis en leurs propres noms.

Cette variable permet d'apprécier la part des sous-traitants qui ont réussi, parallèlement à la sous-traitance, à franchir le niveau de vendeur de produits finis. Aussi, l'inclusion de cette variable est justifiée par le besoin de la présente recherche à identifier les facteurs qui ont permis à ce genre d'entreprises de passer au développement et vente de produits finis. Ce qui renforcera la pertinence et la légitimité des orientations à proposer. Car l'analyse de ces facteurs contribuera à l'identification des bases des orientations et pistes à suivre pour pouvoir passer à ce stade.

Depuis les années 2000, la stratégie des industriels au Maroc consiste à pousser les opérateurs à se reconvertir vers la fabrication du produit fini et de chercher de nouveaux marchés. Donc il s'agit, en d'autres termes, de l'intégration aussi bien en amont qu'en aval. Les entreprises marocaines doivent poursuivre leurs efforts pour intégrer des services en cotraitance tout en conservant leurs clients en sous-traitance. Considérant les exigences concurrentielles, les industriels doivent atteindre le niveau technique et organisationnel exigé pour passer au statut de fabricant de produits finis. La figure suivante expose cette graduation.

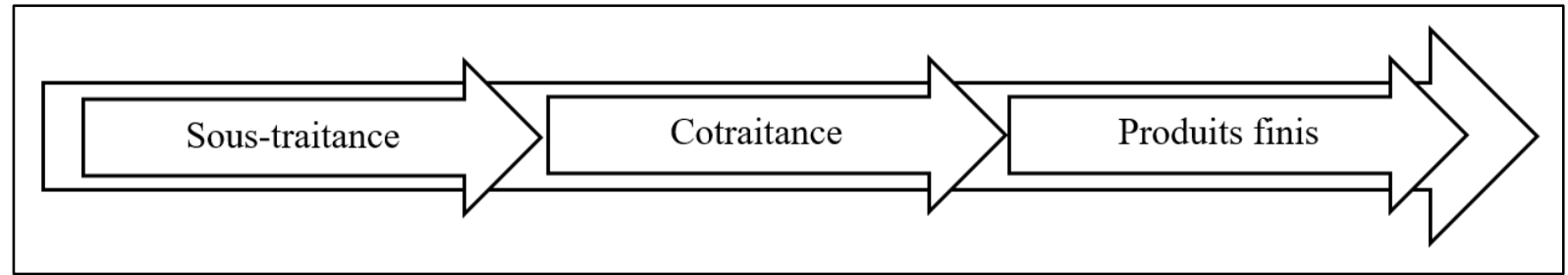

Figure 3 : graduation du degré d'intégration

\section{Hypothèse 1 : le passage de la sous-traitance aux produits finis est résultats d'efforts spécifiques.}

\section{- Part du CA issu de vente de produits finis}

La réussite d'un sous-traitant de sortir de la sous-traitance est matérialisée par sa capacité de développement des produits finis. Toutefois, cette évolution se fait généralement de manière progressive. A cet égard, une analyse de la part du CA issu des ventes de produits finis par rapport au CA global permettra d'apprécier le niveau de conversion. Cette analyse sera plus pertinente en analysant des données sur un fil de temps afin d'essayer d'identifier la corrélation entre l'évolution de cette part et l'évolution des autres variables.

Hypothèse 2 : l'accroissement de la part du chiffre d'affaires issu de la vente de produits finis est un indicateur révélateur du niveau d'intégration du sous-traitant.

- $\quad$ Rang du sous-traitant 
Comme il a été développé dans les chapitres précédents, les sous-traitants sont classés en plusieurs rangs ou niveaux (Kechidi, 2006 ; Benhar, 2016 ; Carrincazeaux et Frigant, 2006 ; Mazaud, 2007 ; Altersohn, 1992 ; Benhar, 2016 ; Ibourk, 2019). Les sous-traitants du premier rang sont les sous-traitants qui travaillent pour le compte du pivot (Mazaud, 2006 ; 2007). Ces sous-traitants peuvent être également appelés dans l'industrie aéronautique et automobile équipementiers ou systémiers (Kechidi, 2006 ; Benhar, 2016 ; Carrincazeaux et Frigant, 2006 ; Mazaud, 2007). Les sous-traitants du deuxième rang sont ceux qui réalisent des activités pour le compte des sous-traitants du premier rang. Ces derniers sont en même temps sous-traitants et donneurs d'ordres. Les sous-traitants du troisième rang sont ceux qui travaillent pour le compte des sous-traitants du deuxième rang.

Considérant les éléments exposés précédemment, il y a une relation entre le type de sous-traitance et le rang du sous-traitant dans le réseau du pivot. A cet effet, on peut admettre que le premier rang est constitué des soustraitants d'intelligence et de fourniture, le deuxième rang est constitué principalement des sous-traitants de spécialité et de technicité et les autres rangs sont constitués généralement des sous-traitants de capacité (Chaillou, 1977; Lainé, 2005; Renou, 2006; Benguerel, 1989; Billaudot et Julien, 2003). Quant à la classification de la sous-traitance en sous-traitance structurelle et sous-traitance conjoncturelle elle est indépendante de la classification précitée (Benguerel, 1989 ; Altersohn, 1992 ; 1997), le critère de classification admis dans ce sens est le temps.

D'un autre côté, cette classification et ce mode organisationnel présente des contraintes pour les entreprises nationales. Car le pivot (entreprise principale) avait déjà son réseau avant d'être implanté au Maroc. Ceci démontre que l'évolution d'une entreprise marocaine d'un niveau à l'autre est résultat de ses efforts à plusieurs niveaux comme le démontre Benabdeljlil et al. (2017) dans le secteur de l'automobile.

Cette variable permet de s'informer sur le niveau qu'occupent la plupart des sous-traitants marocains et comprendre les facteurs qui ont permis, le cas échéant, aux sous-traitants de passer d'un rang à un autre.

Hypothèse 3 : le rang du sous-traitant est un indicateur révélateur du niveau d'intégration du soustraitant.

\section{- Chainons contrôlés}

Si certaines études avancent que la participation des PME dans les chaines de valeurs mondiales est bénéfique pour elles quelle que soit la valeur des activités qu'elles réalisent (OCDE et OMC, 2013), il faudrait noter que la nature et la valeur des chainons comptent beaucoup dans l'intégration des entreprises. C'est la raison pour laquelle les grands donneurs d'ordres se contentent de la réalisation des activités à forte valeur ajoutée, à travers lesquelles elles contrôlent toute la chaine (Perraudin et al., 2014). Donc la nature des activités confiées au sous-traitant peuvent révéler sa compétence et son niveau technique. Aussi, le sous-traitant qui est chargé des produits ou services à forte valeur ajoutée est bien apprécié par le donneur d'ordres et le considère comme acteur capital dans son réseau.

En outre, les sous-traitants qui intègrent des chainons en amont ou en aval peuvent passer à la production de produits finis. Dans ce sens, il convient de noter que l'intégration verticale peut être en amont ou en aval : pour ce qui est de l'intégration en amont, elle conduit l'entreprise à entrer dans une activité qui produit des facteurs et de la matière première qui sont par la suite affectés et incorporés dans son activité d'origine. Elle devient sa propre fournisseur sans fait recours à une autre entreprise. Quant à l'intégration en aval, elle amène l'entreprise à couvrir une activité ou une industrie afin de distribuer ou vendre ses produits ou services. Elle devient son 
propre distributeur. En conséquence, l'intégration verticale garanti davantage le contrôle sur l'ensemble de la chaîne de production d'un produit ou d'un service, ce qui permet d'assurer une certaine sécurité d'approvisionnement, des économies sur l'achat de produits intermédiaires, ou encore indirectement la croissance commerciale de l'entreprise. Cependant, il y a lieu de noter que l'intégration d'activités est question de plusieurs paramètres dont fait partie la valeur et le niveau technologique. Selon Porter (1982), «l'intégration verticale est la combinaison de processus de production, de commercialisation, de vente et/ou processus économiques distincts à l'intérieur des limites d'une seule entreprise... Au lieu d'avoir sa propre force de vente, une firme pourrait avoir passé contrat sur le marché avec une autre organisation de vente indépendante afin d'offrir les services de vente dont elle a besoin $»^{7}$.

Hypothèse 4 : le niveau d'intégration des sous-traitants dépend de la nature des activités qui leur sont confiées.

\section{- Capacité de production}

La capacité de production désigne la capacité effective mensuelle d'un système de production dont dispose un sous-traitant. Donc il s'agit de sa capacité de livraison mensuelle. L'étude de cette variable permettra d'une part de déduire la corrélation entre le degré d'intégration et la capacité de production et d'autre part, de comprendre la manière par laquelle les sous-traitants gèrent leurs capacités étant donné que la revue de littérature démontre que le secteur de textile-habillement est fortement dominé par la sous-traitance de capacité.

Hypothèse 5 : il existe une corrélation positive entre la capacité de production et le niveau d'intégration des sous-traitants.

\section{OPERATIONNALISATION DE LA RECHERCHE}

L'implémentation de la présente recherche a été faite à travers une enquête réalisée par le biais d'un questionnaire envoyé à une population constituée de 176 sociétés. La détermination de ladite population a été faite à travers la collecte d'informations auprès de sources fiables. Ainsi, le traitement des informations collectées a permis, finalement, de traiter des données relatives à un échantillon composé de 44 sociétés, soit $25 \%$ de la population initiale.

\section{A. Répartition géographique}

L'analyse des données collectées à propos des 44 entreprises étudiées a permis de constater que 30 (68\%) sont installées à Casablanca, 6 à Tanger, 3 à Meknès et 1 dans chacune des villes de Fès, Salé, Kenitra, Berrchid et Mohammedia. Ces informations sont récapitulées dans le tableau suivant :

TABLEAU I : REPARTITION GEOGRAPHIQUE DES ENTREPRISES ETUDIEES

\begin{tabular}{|c|c|}
\hline Ville & $\begin{array}{c}\text { Nombre } \\
\text { d'entreprises }\end{array}$ \\
\hline Casablanca & 30 \\
\hline Tanger & 6 \\
\hline
\end{tabular}

${ }^{7}$ Michael E. Porter « Choix stratégique et concurrence », 1982, pp. 328. 


\begin{tabular}{|c|l|}
\hline Meknès & 3 \\
\hline Fès & 1 \\
\hline Salé & 1 \\
\hline Kenitra & 1 \\
\hline Berrchid & 1 \\
\hline Mohammedia & 1 \\
\hline
\end{tabular}

Source : établi par l'auteur

\section{B. Répartition en fonction de la taille}

L'analyse de la taille des entreprises étudiées a permis de constater que 50\% ont un effectif qui varie entre 50 et 250. Quant aux entreprises ayant un effectif dépassant 250, elles représentent 39\%. Ainsi, seulement $11 \%$ ont un effectif inférieur à 50. Le tableau ci-après récapitule ces informations :

TABLEAU II : REPARTITION DES ENTREPRISES ETUDIEES EN FONCTION DE LEUR EFFECTIF

\begin{tabular}{|c|c|}
\hline Effectif & $\begin{array}{c}\text { Nombre } \\
\text { d'entreprises }\end{array}$ \\
\hline$[1-25[$ & 1 \\
\hline$[25-50[$ & 4 \\
\hline$[50-250[$ & 22 \\
\hline$[250-500[$ & 13 \\
\hline$[500-1000[$ & 2 \\
\hline$[1000-5000[$ & 2 \\
\hline
\end{tabular}

Source : établi par l'auteur

\section{Répartition en fonction d'âge}

La majorité des entreprises étudiées ont entre 20 et 30 ans d'existence. Cette catégorie représente $36 \%$ de l'échantillon étudié (16 entreprises). Une seule entreprise a moins de 10 ans d'existence et 7 ont plus de 10 et moins de 20 ans. Il y a lieu de noter que la présente étude a porté sur des entreprises ayant plus de 50 ans, voire plus de 70 ans comme détaillé dans le tableau suivant :

TABLEAU III : REPARTITION DES ENTREPRISES ETUDIEES EN FONCTION DE LEUR AGE

\begin{tabular}{|c|c|c|}
\hline Age & $\begin{array}{c}\text { Nombre } \\
\text { d'entreprises }\end{array}$ & $\%$ \\
\hline$] 1-10]$ & 1 & $2 \%$ \\
\hline$] 10-20]$ & 7 & $16 \%$ \\
\hline$] 20-30]$ & 16 & $36 \%$ \\
\hline$] 30-40]$ & 10 & $23 \%$ \\
\hline$] 40-50]$ & 4 & $9 \%$ \\
\hline$] 50-70]$ & 3 & $7 \%$ \\
\hline$] 70-100]$ & 3 & $7 \%$ \\
\hline
\end{tabular}

Source : établi par l'auteur 


\section{Répartition en fonction du capital}

Le $1 / 4$ des entreprises étudiées ont un capital social qui varie entre 2 et 5 millions de dirhams, et seulement 2 entreprises ont un capital social moins de 2 millions de dirhams. Le classement des entreprises étudiées en fonction de leur capital social est présenté dans le tableau suivant :

TABLEAU IV : REPARTITION DES ENTREPRISES EN FONCTION DE LEUR CAPITAL

\begin{tabular}{|c|c|}
\hline $\begin{array}{c}\text { Capital social en } \\
\text { KMAD }\end{array}$ & $\begin{array}{c}\text { Nombre } \\
\text { d'entreprises }\end{array}$ \\
\hline$[500-1000[$ & 2 \\
\hline$[1000-2000[$ & 7 \\
\hline$[2000-5000[$ & 11 \\
\hline$[5000-10000[$ & 5 \\
\hline$[10000-20000[$ & 9 \\
\hline$[20000-50000[$ & 5 \\
\hline$[50000-100000[$ & 1 \\
\hline$[100000-200000]$ & 4 \\
\hline
\end{tabular}

Source : établi par l'auteur

\section{E. Forme juridique}

Quant à la forme juridique des entreprises étudiées, il convient de noter que $68 \%$ sont des S.A.R.L contre seulement $32 \%$ de S.A.

TABLEAU V : REPARTITION EN FONCTION DE LA FORME JURIDIQUE

\begin{tabular}{|c|c|c|}
\hline $\begin{array}{c}\text { Forme } \\
\text { juridique }\end{array}$ & $\begin{array}{c}\text { Nombre } \\
\text { d'entreprises }\end{array}$ & $\mathbf{\%}$ \\
\hline S.A & 14 & 32 \\
\hline S.A.R.L & 30 & 68 \\
\hline
\end{tabular}

Source : établi par l'auteur

\section{RESULTATS ET DISCUSSION}

Les résultats présentés dans ce chapitre ont été dégagés à travers une étude portée sur l'échantillon de soustraitants marocains présenté en haut. Ces résultats sont détaillés dans les sections suivantes.

\section{A. Niveau d'intégration : un secteur majoritairement dominé par la sous-traitance}

L’intégration évoquée dans cette étude veut que les entreprises doivent acquérir une expérience (savoir-faire, compétences techniques et technologie nécessaire) dans la transformation des matières premières en produits finis dans des délais très courts. Aussi, la valeur ajoutée des entreprises réside dans leur productivité, leur réactivité et la performance de leurs outils de production.

L'analyse des résultats présentés dans la figure 4 permet de conclure que peu d'entreprise dans ce secteur ont réussi à développer leur niveau d'intégration en passant de la simple sous-traitance au développement de produits finis en leurs propres noms. Bien que le nombre de ces entreprises demeure faible, il convient de noter 
que l'étude des facteurs de leur 'succès" permettra de déduire les pistes d'amélioration du niveau d'intégration des autres sous-traitants.

En effet, la majorité des firmes opérant dans le secteur du textile-habillement sont des sous-traitants et leur capacité de production avoisine dans la majorité des cas 100000 pièces par mois. La cartographie présentée dans la figure suivante permet de relater ces constats en se basant sur deux variables, à savoir : le degré d'intégration et la capacité de production mensuelle.

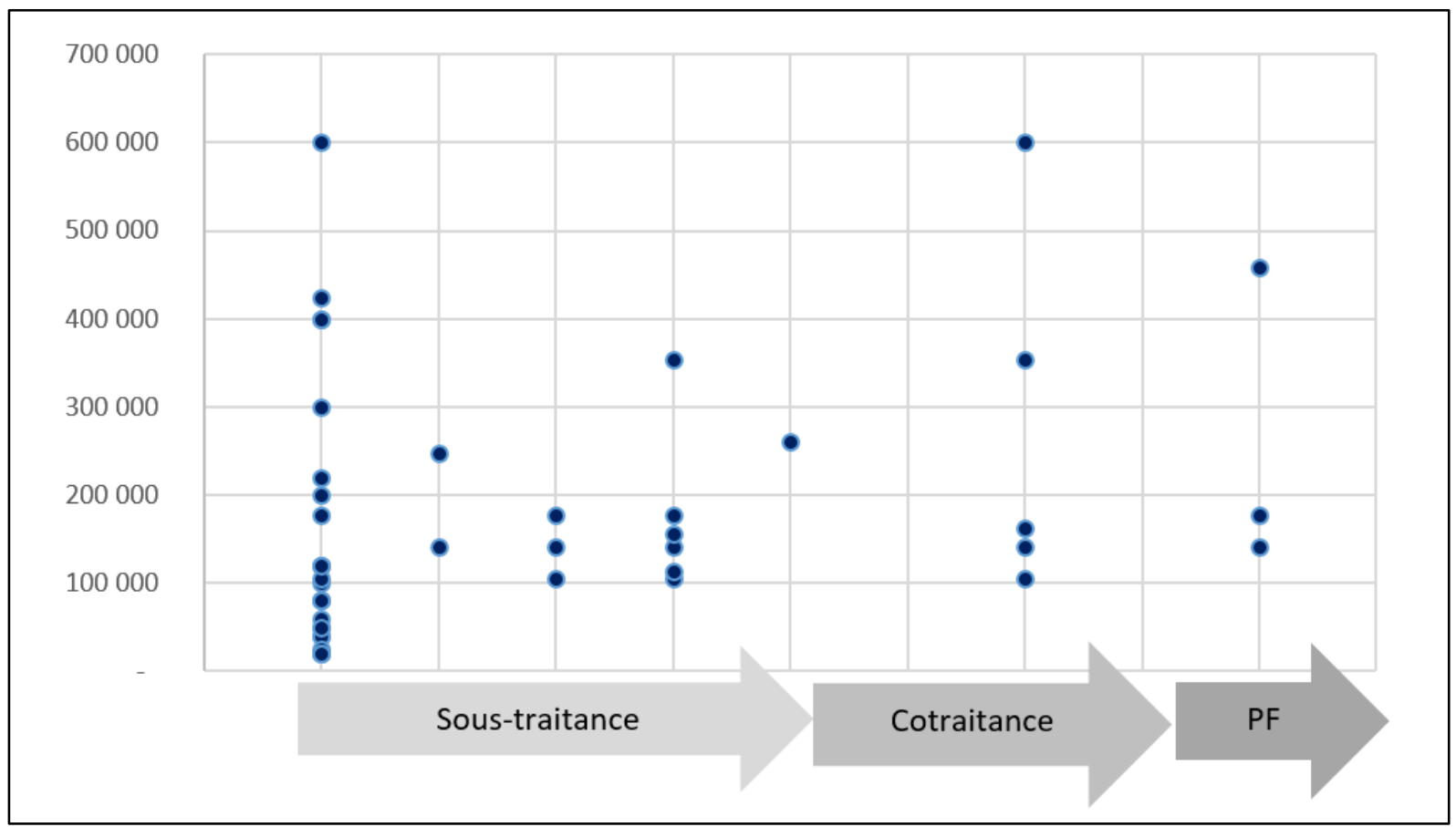

Figure 4 : degré d'intégration des sous-traitants dans le secteur du textile et leurs capacités

L'analyse du degré d'intégration des sous-traitants marocains dans le secteur du textile en fonction de leurs capacités de production a permis de constater qu'ils peuvent être classés en 5 catégories. Cette classification est obtenue en adoptant trois variables à savoir : le degré d'intégration, la capacité de production et la nationalité des donneurs d'ordres.

La première catégorie se compose des sous-traitants disposant d'une capacité de production faible et d'une main d'œuvre moins qualifiée. Ces sous-traitants sont spécialisés dans certaines étapes découlant de chaines de valeurs contrôlées par des donneurs d'ordres marocains. Ces derniers sont à leur tour des sous-traitants de donneurs d'ordres étrangers.

La deuxième catégorie est composée de sous-traitants disposant d'une capacité de production qui varie entre 100000 et 300000 pièces par mois. Ces sous-traitants se chargent dans la majorité des cas de la confection qui est une étape relevant d'une chaine de valeurs contrôlée par des grands donneurs d'ordres. La majorité des acteurs dans le secteur du textile-habillement au Maroc peuvent être classés dans ces deux premières catégories.

La troisième catégorie est composée des sous-traitants qui ont passé à la cotraitance. Les acteurs classés dans cette catégorie disposent de capacités de production qui varient entre 100000 et 600000 pièces par mois.

La quatrième catégorie est composée des entreprises qui disposent d'une capacité de production importante mais restent toujours dans le niveau de sous-traitance. Ces acteurs opèrent avec plusieurs donneurs d'ordres, notamment des donneurs d'ordres européens, qui sont reconnus à l'échelle mondiale. 
Quant à la cinquième catégorie, elle contient des entreprises marocaines qui ont réussi à développer leurs niveaux d'intégration en multipliant leurs offres. Ces acteurs ont pu diversifier leurs clientèles en franchissant le niveau de produits finis. Ces firmes ont étendu leurs plateformes de production en développant leur chaine de production.

Le pourcentage de chaque catégorie dans l'échantillon étudié dans cette recherche est présenté dans la figure ci-après :

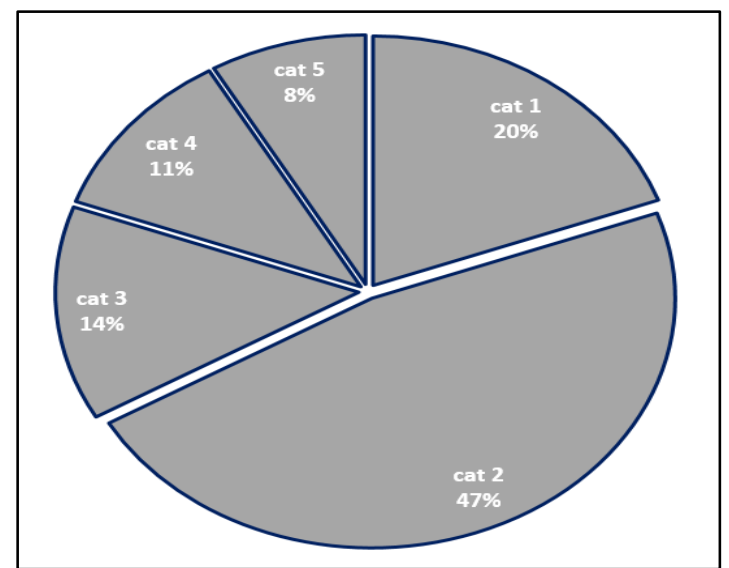

Figure 5 : catégorisation des entreprises étudiées

Il ressort de cette figure que la majorité d'acteurs sont des sous-traitants et faiblement équipés. En effet, $78 \%$ des entreprises couvertes par cette enquête sont toujours dans la sous-traitance. Dont presque 2/3 disposent d'une capacité de production inférieure à 200000 unités par mois.

\section{B. La capacité de production et le niveau d'intégration}

La revue de littérature exposée en haut mène à s'interroger sur la capacité de production et son rôle dans l'intégration des entreprises marocaines. A cet égard, il va sans dire que le sous-traitant doit disposer d'une capacité suffisante pour pouvoir réaliser les activités dont il a la charge dans les délais. Toutefois, certains soustraitants, notamment ceux travaillant avec des clients de renom international, ont construit autour d'eux des écosystèmes locaux (réseaux) composés de sous-traitants et fournisseurs nationaux et étrangers. Ainsi, l'entreprise principale constitue une locomotive tirant derrière-elle tout un écosystème qui lui permet de réaliser les commandes dans les délais et conformément aux exigences prédéfinies. Ceci signifie que même si le soustraitant ne dispose pas de la capacité de production nécessaire pour la réalisation des commandes qu'il reçoit, il recourt à son tour à la sous-traitance nationale, voire certaines sous-traitant ne dispose que d'un petit centre dédier aux études, d'une entité chargée du suivi des sous-traitants et des autres fonctions support, et que toutes les autres tâches de production et de transformation sont assurées par les sous-traitants. De même, dans le cadre de cette enquête, il a été constaté que la sous-traitance est assurée dans certains cas par des entreprises relevant du groupe auquel fait partie le donneur d'ordres (sous-traitant principal). Dans d'autres cas, le donneur d'ordres est l'actionnaire majoritaire dans ses sous-traitants. Cette organisation est justifiée par des raisons organisationnelles, juridiques et fiscales.

Pour ce qui est de la stabilité du portefeuille de fournisseurs, il convient de préciser que la majorité de ces entreprises disposent d'un portefeuille de sous-traitants qui travaillent à $100 \%$ avec elles. Donc il s'agit de sociétés crées pour cet effet dont les capitaux sont détenus par les donneurs d'ordres (sociétés mères). Dans certains cas, les sous-traitants sont installés dans le même local que le donneur d'ordres. De même, il y a lieu 
de signaler que les activités et tâches sous-traitées sont normales et ne nécessitent pas un niveau de savoir spécifique. Aussi, il convient de noter que dans la majorité des cas similaires à cette organisation plus de $75 \%$ de l'effectif global est employé dans la production, moins de $10 \%$ dans la $R \& D$, contre $4 \%$ dans la direction financière.

Cette organisation peut être de manière séquentielle, c'est-à-dire que chaque sous-traitant s'occupe d'une partie dans la chaine de production comme le montre la figure suivante :

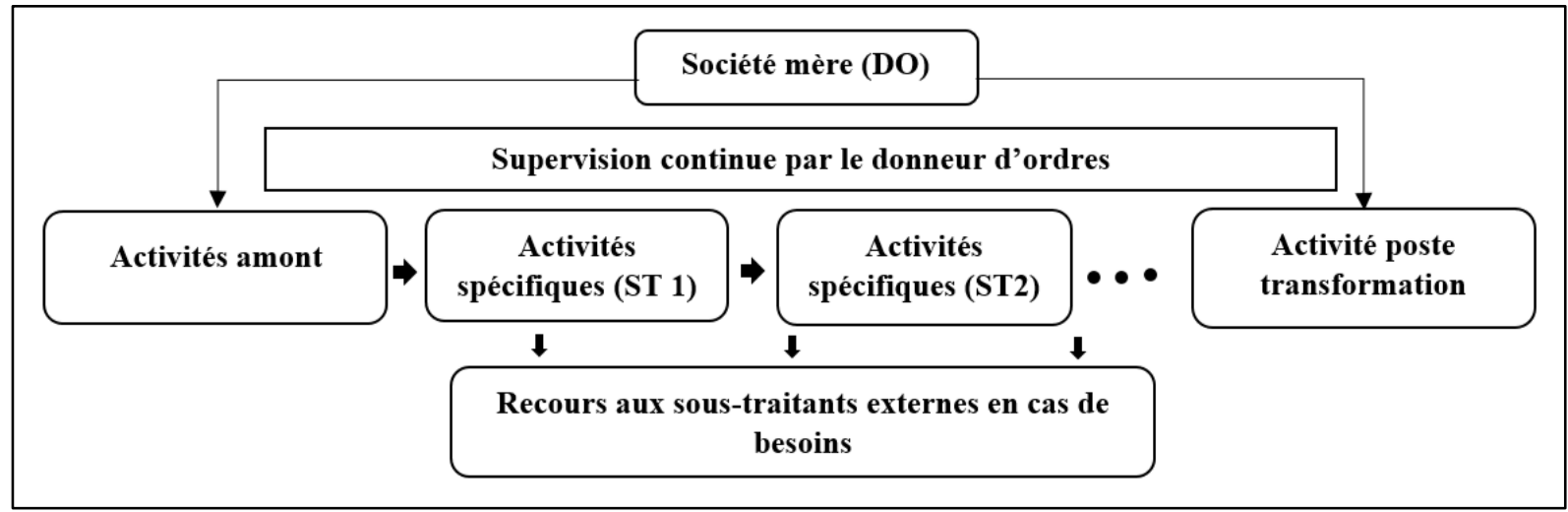

Figure 6 : organisation séquentielle

En plus de cette organisation, il existe une deuxième organisation dite parallèle. Cette organisation impose que les sous-traitants doivent être dotés des mêmes outils de production surtout lorsqu'ils s'occupent du même produit.

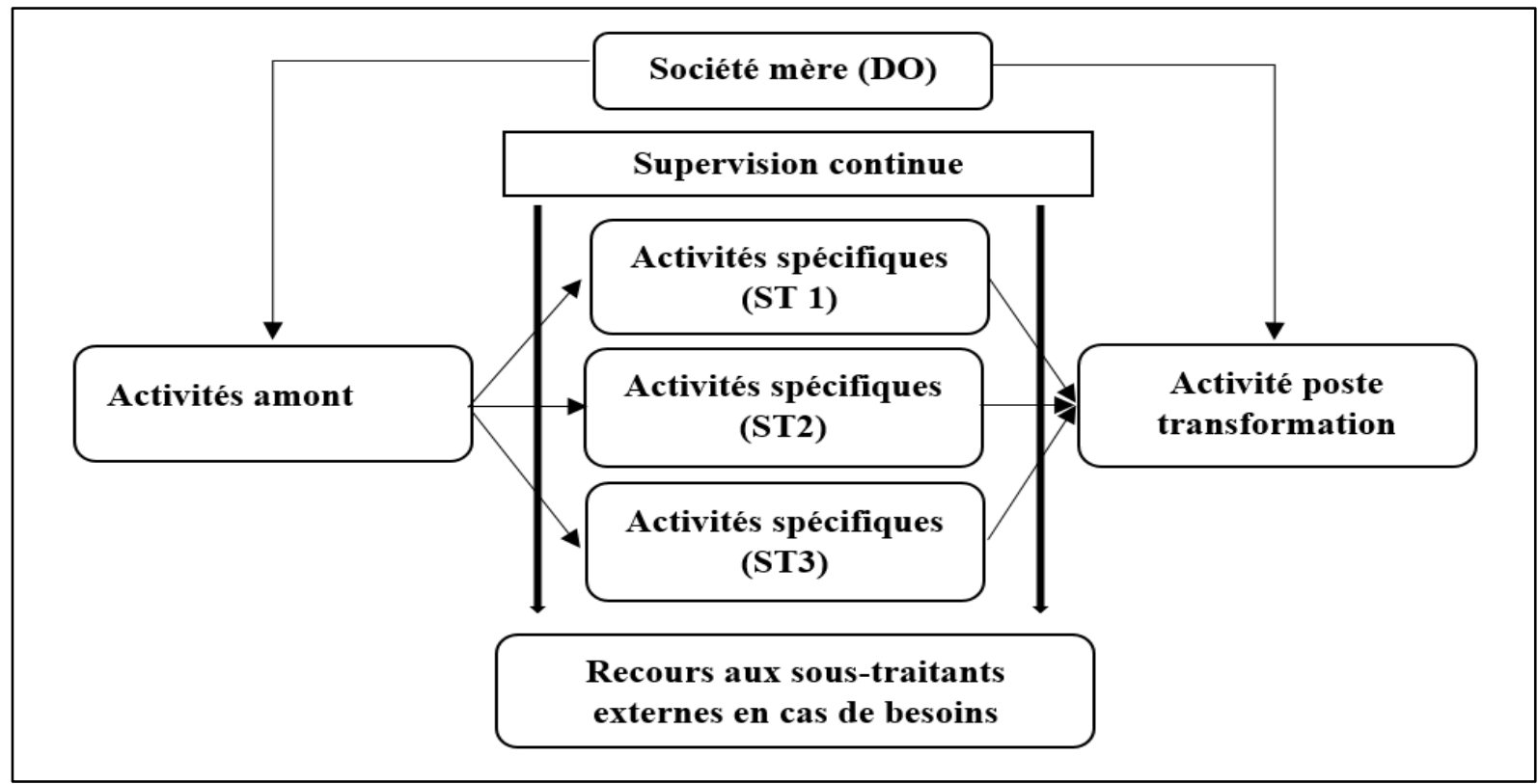

Figure 7 : organisation parallèle

Il est opportun de s'interroger sur la pertinence de ces organisations au lieu d'une seule société dont les filiales sont érigées en divisions ou départements internes. L'analyse menée à ce propos a permis de constater que ces organisations présentent les avantages suivants :

- Diversification des risques ; 
- Avantages fiscaux ;

- Bénéfice des aides directes de l’Etat ;

- Efficacité de contrôle.

Dans ce cas, les principaux rôles de la société mère, qui est en même temps donneuse et preneuse d'ordres, peuvent être résumés suivant :

- Le développement de nouvelles collections et modèles à proposer aux clients. En effet, le maillon de la chaine relatif au développent qui sont confiées aux entités appelées généralement « bureau d'études ou $\mathrm{R} \& \mathrm{D}$ » est logé au niveau de la société mère (donneur d'ordres) ;

- La prospection des clients et donneurs d'ordres et ce, généralement à travers des entités (directions, divisions, services...) chargées de la gestion des aspects marketing et commerciales ;

- L'approvisionnement en matières premières et accessoires. Etant donné que l'approvisionnement en matières premières et accessoires ne peut s'effectuer en excluant les entités chargées de la production, le donneur d'ordres dispose d'une entité chargée du suivi des sous-traitants, quand ils relèvent du même groupe, ou avec coordination directe avec les sous-traitants qui définissent et déterminent leurs besoins en matières premières et fournitures ;

- Le suivi de la logistique des matières premières et fourniture, le choix des sous-traitants, la fixation des plannings avec les sous-traitants et la supervision des sous-traitants.

Pour ce qui est des outils informatiques utilisés par les entreprises étudiées dans leurs processus métiers et supports, ils sont généralement : SAGE, MODAPRO, LECTRA, CERBER, OPTITIME, ALPHA CSD, etc.

Bien qu'il ait été annoncé que les maillons d'amont ont été renforcés, notamment les producteurs locaux de matières premières, et ce, pour réduire significativement les délais de livraison, cette enquête a permis de constater que la majorité des matières premières sont importées. De même, il a été révélé que dans plusieurs cas, ce sont les donneurs d'ordres qui achètent les matières premières. Ce qui signifie que le choix des matières premières et la sélection des fournisseurs se font généralement par les donneurs d'ordres.

\section{Sous-traitants faisant partie de réseaux de DO étrangers}

On appelle chaine de valeur l'ensemble des activités requises, de la conception à la production, jusqu'à la livraison du produit fini au consommateur final, et éventuellement, son recyclage. Elle retrace l'ensemble des étapes du processus d'offre (conception, acquisition des inputs, transformation, marketing, et vente, services après-vente et recyclage).

Dans le cas particulier du textile-habillement, les différents segments de la chaine de valeur sont: la production des fibres, l'égrenage, la filature et le tissage, le tricotage, l'impression, la confection, la vente au détail. Les organisations de distribution de détail basées dans les pays de consommation, dominent complètement la filière. Leur force réside dans la collecte des informations liées au marché, en partant des tendances les plus récentes sur les styles et les goûts.

La majorité des entreprises nationales se positionnent sur le segment de la confection. Elles assurent la réception des modèles de ses donneurs d'ordres, ainsi que les tissus, et assurent l'approvisionnement des fils et accessoires, la confection et le transport. Cette catégorie de sociétés sont tenues de respecter les exigences de production (transformation de produits) et les délais de livraison. La performance de cette catégorie réside dans sa productivité et sa réactivité et la performance de son outil de production. 


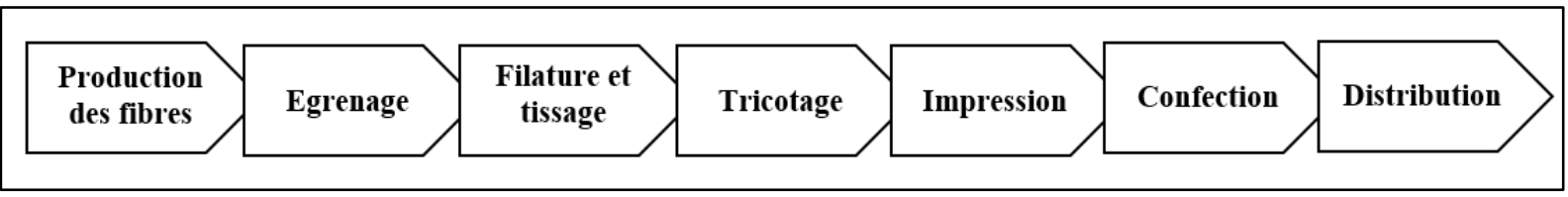

Figure 8 : chaine de valeur de la plupart des entreprises marocaines

Le maillon «Confection » se compose des activités liées à la réception des commandes, la planification de la production et l'approvisionnement en accessoires et fourniture, la coupe, la façon, l'emballage et la logistique. Il peut être ventilé en sous maillons comme schématisé dans la figure suivante :

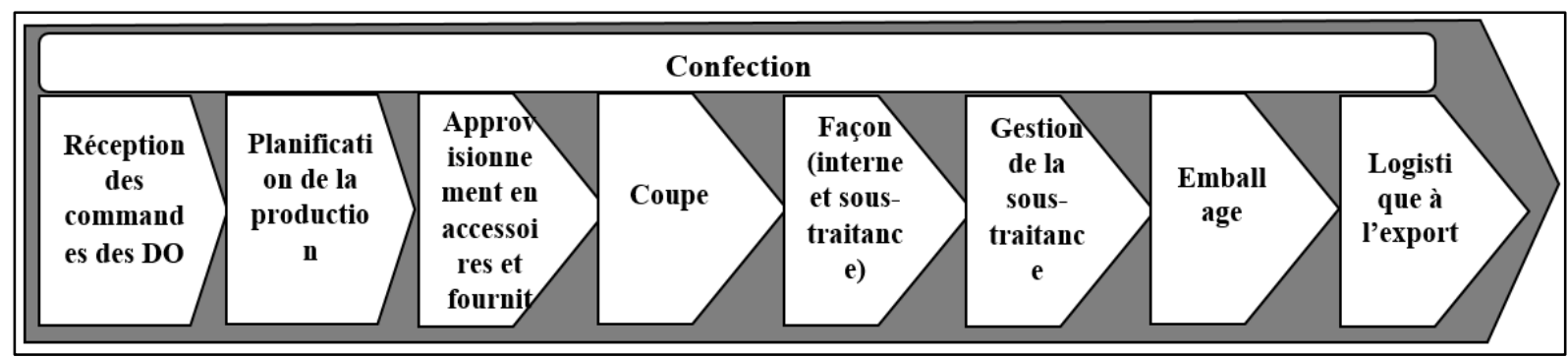

Figure 9 : décomposition de l'activité de confection

Pour ce qui est de l'étape «Façon» elle peut être faite en interne comme elle peut être sous-traitée. La présente enquête a permis de constater que certaines entreprises sous-traitent jusqu'à $20 \%$ de cette activité.

\section{Sous-traitants acteurs d'écosystèmes nationaux}

Cette enquête a permis de constater, aussi, l'existence de sous-traitants dont les clients sont des entreprises nationales. Ces dernières fabriquent des produits finis en leurs propres noms, mais elles sont peu par rapport à l'autre catégorie qui contient des sous-traitants du premier et deuxième niveau. Cette catégorie de sous-traitants se caractérise principalement par :

- la faiblesse de leurs capacités de production ;

- la simplicité de leurs systèmes de production ;

- la main d'œuvre moins qualifiée ;

- La majorité est concentrée sur l'étape relative au façonnage ;

Par ailleurs, cette enquête a permis de relevé une observation impressionnante à propos de la capacité de production et le développement des produits. En effet, nous avons cru avant que les entreprises fabricant des produits finis disposent de capacités de production plus élevées. Toutefois, il convient de noter que la capacité de production n'est pas un critère déterminant dans le passage au niveau de produits finis. Par conséquent, une question importante s'impose à savoir : quels sont les facteurs clés de succès de ces entreprises ?

Les entreprises de cette catégorie, qui se distinguent de la majorité des entreprises du secteur, proposent une large offre de service et ne sont pas spécialisées dans les produits finis. Elles proposent des offres de soustraitance et de cotraitance et produisent en même temps en leurs propres noms. En analysant les informations recueillies par cette enquête, il a été constaté que cette catégorie réalise des chiffres d'affaires qui sont dans certains cas huit fois supérieurs au chiffre d'affaires moyen du secteur. Toutefois, le chiffre d'affaires émanant des ventes de produits finis ne dépasse pas actuellement $1 / 4$ (25\%) de leur chiffre d'affaires global. Certaines entreprises l'estiment évoluer à plus de $40 \%$ dans les cinq prochaines années. Ces entreprises ont réussi à se 
démarquer de la majorité des entreprises nationales qui sont majoritairement dans la sous-traitance grâce aux facteurs suivants :

- Le lancement dès leur démarrage dans une politique de développement de produits finis, ainsi, la distinction s'est adoptée dans leur politique de management et de gestion ;

- Le développement d'un portefeuille client important, marqué par des donneurs d'ordres reconnu à l'échelle mondiale ;

- Le ciblage de nouvelles niches ;

- La pénétration de nouveaux marchés.

E. Intégration d'une chaine de valeur dominée par le groupe

Le sous-traitant de grands donneurs d'ordres étrangers est une société « holding » qui détient la majorité ou la totalité des capitaux de plusieurs filiales tel qu'illustré dans les figures 6 et 7 . Il détient la majorité des capitaux de plusieurs autres entreprises qui sont leurs principaux fournisseurs. Après la conclusion d'accords avec les donneurs d'ordre étrangers, l'entreprise principale (société mère) achète les matières premières nécessaires pour la production des produits demandés par les donneurs d'ordres. Elle ordonne ensuite à ses filiales de procéder à la fabrication des composants nécessaires à la fabrication des produits commandés par les donneurs d'ordres.

Ces sous-traitants ont bien intégré la chaine de valeur de production à cause des éléments suivant :

- Forte implication du DO : étant donné que toutes les entreprises concernées par cette chaine de valeur confrontent les même enjeux. Autrement dit, les défis qui leur sont imposés par la concurrence les poussent à respecter les exigences des donneurs d'ordres notamment en matière de qualité et de délais. C'est les raisons pour lesquelles la société mère s'implique dans toutes les étapes de production des composantes du produit sous-traité. Cette implication du donneur, représente pour les sous-traitants une occasion pour développer leurs compétences. De même, les sociétés mères, qui sont à leurs tours des sous-traitants de grands donneurs d'ordres étrangers, apportent un appui technique, managérial et financier permettant aux sous-traitants de gérer leurs problèmes. Donc, il s'agit d'une assistance et un accompagnement continu ;

- Transfert de technologie assuré : dans certains cas, toute la valeur ajoutée dégagée au niveau de la chaine de valeur est réalisée par les sous-traitants (filiales). Par conséquent, la société mère doit appuyer leur compétitivité.

\section{F. Intégration par un seul donneur d'ordre}

En examinant les données constituées concernant l'échantillon des sous-traitants étudiés dans le cadre de ce travail, il a été constaté que certains sous-traitants ont conclu des contrats de sous-traitance avec un seul donneur d'ordres. En effet, le sous-traitant peut travailler en exclusivité pour un seul donneur d'ordres. Cette catégorie, exerçant toutes ses activités avec un seul donneur d'ordres, bénéficie de certains avantages qu'offre cette situation. En effet, l'exclusivité des ventes à un seul donneur d'ordres peut engendrer la stabilité du chiffre d'affaires du sous-traitant, au moins sur une période qui peut dépasser cinq ans. Cette période est considérée comme importante vue les mutations économiques et les bouleversements de la chaine de valeurs mondiale. En effet, pour une entreprise qui maitrise ses ventes sur une période plus au moins importante, ceci est considéré comme avantage.

Considérant la stabilité du chiffre d'affaires, les entreprises peuvent entamer des ajustements en ce qui concerne les composantes des coûts de revient. Ainsi, de cette façon, les sous-traitants procèdent au 
remaniement des composantes du coût tout en gardant la même qualité et en respectant les délais de livraison. A cet égard, la stabilité du chiffre d'affaires est considérée par les entreprises comme facteur clé pour repenser les mécanismes d'efficacité.

En revanche l'implication du donneur d'ordre n'est pas toujours bénéfique pour les sous-traitants notamment à long terme. Du fait qu'elle pourrait rendre de ses derniers des simples façonniers qui vendent de la main d'œuvre. Ce genre d'implication est fortement observé dans le secteur du textile habillement. La majorité des donneurs d'ordre dans ce secteur sont en même temps fournisseurs et clients. En effet, les donneurs d'ordre impose à leurs fournisseurs les méthodes de travail et leur fournit les matières premières. Autrement-dit, le donneur d'ordre s'occupe de l'approvisionnement (tissus, fils, accessoires,...) et de la spécification des méthodes de travail (modèles) selon un cahier de charges. Il y a donc une relation de subordination où le soustraitant est un simple vendeur de main d'œuvre. Pour certains sous-traitants, cette situation en plus qu'elle lui garantit une sources de revenu en s'adaptant aux besoins du donneur d'ordres, elle peut être considérée comme canal de transfert de technologie et de savoir-faire et ce, à travers la formation des salariés pour pouvoir utiliser les machines dont l'utilisation nécessite à l'acquisition de certaines compétences et techniques, la délocalisation de certains usines, l'emploi, etc.

Du à la mondialisation et aux bouleversements de l'environnement, aujourd'hui les donneurs d'ordres exercent une forte pression sur les sous-traitants notamment en matière de prix et de qualité. En effet, les soustraitants doivent rester en mesure de veiller sur l'environnement externe notamment en ce qui concerne les technologies adoptées et les méthodes et procédures de production permettant de produire des biens de qualité avec moindres coûts.

\section{CONCLUSION}

L'amont et l'aval d'une chaine de valeur sont généralement trop contrôlés par le pivot (l'entreprise principale). Car, c'est là où se trouvent les chainons à forte valeur ajoutée et les clefs de la dominance. L'amont se compose des étapes de conception et de développement des produits et services et l'aval se compose des chainons de commercialisation et de développement de l'image de l'entreprise. Par conséquent, le contrôle de ce genre d'activités est indispensable pour la survie de n'importe quel pivot. C'est pourquoi, les grands donneurs d'ordre s'occupent souvent eux-mêmes des chainons en amont ou les délèguent à d'autres entreprises disposant de caractéristiques techniques dépassant les leur.

Il va sans dire que le sous-traitant doit disposer d'une capacité suffisante pour pouvoir réaliser les activités dont il a la charge dans les délais. Toutefois, certains sous-traitants, notamment ceux travaillant avec des clients de renom international, ont construit autour d'eux des écosystèmes locaux (réseaux) composés de sous-traitants et fournisseurs nationaux et étrangers. Ainsi, l'entreprise principale constitue une locomotive tirant derrière-elle tout un écosystème qui lui permet de réaliser les commandes dans les délais et conformément aux exigences prédéfinies. Ceci signifie que même si le sous-traitant ne dispose pas de la capacité de production nécessaire pour la réalisation des commandes qu'il reçoit, il recourt à son tour à la sous-traitance nationale, voire certaines sous-traitant ne dispose que d'un petit centre dédier aux études, d'une entité chargée du suivi des sous-traitants et des autres fonctions support, et que toutes les autres tâches de production et de transformation sont assurées par les sous-traitants. De même, dans le cadre de cette enquête, il a été constaté que la sous-traitance est assurée dans certains cas par des entreprises relevant du groupe auquel fait partie le donneur d'ordres (sous-traitant principal). Dans d'autres cas, le donneur d'ordres est l'actionnaire majoritaire dans ses sous-traitants. Cette organisation est justifiée par des raisons organisationnelles, juridiques et fiscales.

Pour ce qui est de la stabilité du portefeuille de fournisseurs, il convient de préciser que la majorité de ces entreprises disposent d'un portefeuille de sous-traitants qui travaillent à $100 \%$ avec elles. Donc il s'agit de 
sociétés crées pour cet effet dont les capitaux sont détenus par les donneurs d'ordres (sociétés mères). Dans certains cas, les sous-traitants sont installés dans le même local que le donneur d'ordres. De même, il y a lieu de signaler que les activités et tâches sous-traitées sont normales et ne nécessitent pas un niveau de savoir spécifique. Aussi, il convient de noter que dans la majorité des cas plus de $75 \%$ de l'effectif global est employé dans la production, moins de $10 \%$ dans la $\mathrm{R} \& \mathrm{D}$, contre $4 \%$ dans la direction financière.

Par ailleurs, les entreprises ayant réussi à développer des produits finis en leurs propres noms, et qui se distinguent de la majorité des entreprises du secteur, proposent une large offre de service et ne sont pas spécialisées dans les produits finis. Elles proposent des offres de sous-traitance et de cotraitance et produisent en même temps en leurs propres noms. En analysant les informations recueillies par cette enquête, il a été constaté que cette catégorie réalise des chiffres d'affaires qui sont dans certains cas huit fois supérieurs au chiffre d'affaires moyen du secteur. Toutefois, le chiffre d'affaires émanant des ventes de produits finis ne dépasse pas actuellement $1 / 4(25 \%)$ de leur chiffre d'affaires global. Certaines entreprises l'estiment évoluer à plus de $40 \%$ dans les cinq prochaines années. Ces entreprises ont réussi à se démarquer de la majorité des entreprises nationales qui sont majoritairement dans la sous-traitance grâce aux facteurs suivants : (1) le lancement dès leur démarrage dans une politique de développement de produits finis, ainsi, la distinction s'est adoptée dans leur politique de management et de gestion, (2) le développement d'un portefeuille client important, marqué par des donneurs d'ordres reconnu à l'échelle mondiale, (3) le ciblage de nouvelles niches et (4) la pénétration de nouveaux marchés.

\section{REFERENCES}

[1] Claude ALTERSOHN, "de la sous-traitance au partenariat industriel, de la piétaille à l'infanterie", Editions l'Harmattan, 1992.

[2] Claude ALTERSOHN, "la sous-traitance à l'aube du XXIe siècle", Editions l'Harmattan, 1997.

[3] La nouvelle tribune, "l'attractivité du Maroc, objectif principal de l'AMDIE", la nouvelle tribune, spécial été 2019, hors série, pp.26-28.

[4] Gilbert Ammar et Nathalie Roux, "Délocalisation et nouveau modèle économique : le cas du secteur textile-habillement", La Revue de l'Ires, 2009/3 nº 62, pp.99 à 134.

[5] Halima BAKALA, "La politique des clusters au Maroc quel avantage pour le secteur de textile-habillement", Revue Interdisciplinaire, Vol1, $\mathrm{n}^{\circ} 3,2017$.

[6] Bernard Baudry, "Quasi-intégration et relation de sous-traitance industrielle : une évaluation des travaux de Jacques Houssiaux », Revue d'économie industrielle, 142. 2e trimestre 2013.

[7] Saâd Belghazi, "Pratiques des donneurs d'ordre et précarité du travail dans le textile habillement marocain", INSEA, Rabat, 2005.

[8] Nadia Benabdeljlil, “apprendre dans un réseau : le cas inédit d'un fournisseur automobile marocain”, Annales des Mines - Gérer et comprendre, $\mathrm{N}^{\circ} 112$, p. 55 à 65, 2013.

[9] Nadia Benabdeljlil et al., "L’émergence d'un pôle automobile à Tanger", Critique économique n 35 • Hiver-printemps 2017.

[10] Jacques Benguerel, “Stratégie des R M. I sous-traitantes”, imprimerie Typofeset Dynamic SA, la chaux-de-fonds, 1989.

[11] Zakaria BENHAR, "les déterminants de la performance des sous-traitants aéronautiques marocains dans la région du grand Casablanca”, thèse soutenue à l'ISCAE, juin 2016.

[12] Bernard Billaudot et Christian Genthon, "les nouvelles formes de coordination industrielle : réexamen du couple marche / hiérarchie et application au cas de l'informatique”, Colloque Histoire, Gestion, Organisations : Marché(s) et hiérarchie(s). 29 et 30 novembre 2001.

[13] Bernard Billaudot et Pierre Julien, “Organisation industrielle et sous-traitance : du fordisme à l'entreprise réseau”, presse de l'université de Québec, 2003.

[14] Christophe Carrincazeaux et Vincent Frigant. "L'internationalisation de l'industrie aérospatiale française durant les années 90. La Décennie de la rupture?”. Cahiers du Gres, n²006-15. 
[15] Mohamed E. Chaffai et Patrick Plane, “Contraintes de financement et productivité dans l'industrie de l'habillement au Maroc en présence d'hétérogénéité de la technologie”, Revue d'économie du développement, 2015/2 Vol. 23, pp. 103 à 132.

[16] Bernard Chaillou, "Définition et typologie de la sous-traitance”, Revue économique, volume 28, n², 1977. pp. $262-285$.

[17] Cécilia Clerwall et al., "Donneurs d'ordres - Sous-traitants: Quels enjeux et quels rôles de la fonction RH pour une relation durable et performante?", mémoire d'expertise, Université Paris-Dauphine, 28 octobre 2013.

[18] Amine Dafir et Ghizlane Salam, "L'intégration de la diplomatie économique dans un dispositif national d'intelligence économique : quels enjeux pour le Maroc ?”, Revue internationale d'intelligence économique, 2016/2 Vol. 8, pp. 47-63.

[19] Anas Httabou et Abdenbi Louitri, "Développement durable et management des PME : une analyse en termes de proximité. illustration par un cas du secteur textile-habillement”, Management Prospective Ed, Management \& Avenir, 2011/3n ${ }^{\circ} 43$, pp : 122 à 142.

[20] Philippe Hugon, "La chine en Afrique, néocolonialisme ou opportunités pour le développement ?", Revue internationale et stratégique, $2008 / 4 \mathrm{n}^{\circ} 72$, pp. 219 à 230.

[21] Aomar Ibourk, "Etude sur le commerce et les chaines de valeur dans les activités porteuses d'emplois (TRAVERA) : cas du secteur automobile au Maroc", BIT, 2019.

[22] Mohamed Kechidi, "Dynamiques de relations verticales dans l'industrie aéronautique : une analyse de la sous-traitance d'Airbus", Cahiers du GRES, n 2006-10.

[23] KHALLAF Nezha, "La relation de coordination entre les agents économiques : Risque ou confiance. Le cas de la sous-traitance dans le textile-habillement au Maroc", thèse soutenue à l’Université d'Artois, Mai 2009.

[24] Frédéric Mazaud, "De la firme sous-traitante de premier rang à la firme pivot Une mutation de l'organisation du système productif Airbus", Revue d'économie industrielle, 113/1er trimestre 2006.

[25] Frédéric MAZAUD, “de la firme sous-traitante de premier rang à la firme pivot, l'organisation du système productif Airbus", thèse soutenue à l'Université des sciences sociales Toulouse I, juillet 2007.

[26] Riad Meddeb, "L'industrie du textile-habillement au Maroc : les besoins des chefs d'entreprise et les conditions des femmes dans les PME", Bureau international du Travail, Genève, Août 2000.

[27] Mihoub Mezouaghi et Karim El Aynaoui, “l'Afrique sur la voie de l'industrialisation ? Économie politique et trajectoires historiques. Introduction thématique”, Afrique contemporaine, $\mathrm{N}^{\circ} 266$, p.11 à 27, 2018.

[28] OCDE et OMC, "Panorama de l'aide pour le commerce 2013 : se connecter aux chaînes de valeur”, OCDE et OMC 2013.

[29] OCDE, “économies interconnectées : comment tirer parti des chaines de valeur mondiales”, OCDE, Paris, 2013.

[30] Corinne Perraudin et al., "Les rapports de force au coeur des relations de sous-traitance : conséquences sur les relations de travail”, Documents de Travail du Centre d'Economie de la Sorbonne - 2014.89, 2014.

[31] Alain Piveteau, “Intégration productive transfrontalière ou reproduction d'un rapport de sous-traitance. Les confectionneurs tangérois", Critique économique n² 25, automne 2009, pp. 17-39.

[32] Michaël E. Porter, “Choix stratégique et concurrence”, 1982, pp. 328.

[33] Yvan Renou, “L’espace de coordination : une nouvelle modalité d'intégration des activités au sein de l'entreprise-réseau. Théorie et illustration des pratiques de co-conception au sein des configurations modernes", lien : https://www.researchgate.net/publication/5084074

[34] Étienne St-Jean et Luc LeBel, "La dépendance commerciale et l'autonomie décisionnelle influencent-elles la performance et les choix stratégiques? Le cas des PME sous-traitantes en opérations forestières, Revue internationale P.M.E., 23 (3-4), p. 95-121, 2010.

[35] World Bank, "Kingdom of Morocco Country Economic Memorandum Fostering Higher Growth and Employment with Productive Diversification and Competitiveness", Report No. 32948, 2 vol., Washington, 2006. 